\title{
Estimates for first exit times of non-Markovian Itô processes
}

\author{
Nikolai Dokuchaev*
}

May 18, 2014

\begin{abstract}
First exit times and their path-wise dependence on trajectories are studied for nonMarkovian Itô processes. Estimates of distances between two exit times are obtained. In particular, it follows that first exit times of two Itô processes are close if their trajectories are close.

Key words: Itô processes, first exit times, non-Markovian, path-wise properties.

MSC 2000 subject classifications: 60G17, 60G40, 60J50, 60J65 60H10

Abbreviated title: Estimates for first exit times of Itô processes
\end{abstract}

\section{Introduction}

First exit times for dynamic process are presented in many problems in stochastic control, optimal stopping, statistical estimation, and mathematical finance. There are classical representation results that connect the probability distributions of first exit times of diffusion processes with the boundary value problems for Kolmogorov parabolic equations. In contrast, the pathwise properties of first exit times are not very well studied. It is easy to see that first exit times from a region for smooth processes do not depend continuously on variations of the initial data or the coefficients. For instance, first exit time from the interval $(-1,1)$ of the process $y(t)=a+\sin t$ does not depend continuously on $a$ near zero. However, first exit times for non-smooth trajectories of diffusion processes have some path-wise regularity with respect to these variations (see Dokuchaev [1]).

In the present paper, the research started in [1] is extended on non-Markov processes. The cited paper studied path-wise dependence on trajectories for first exit times from a

*Department of Mathematics, Trent University, Ontario, Canada

Published in Stochastics 80 (2008), 397-406. 
bounded domain $D \subset \mathbf{R}^{n}$ for Markov diffusion processes. The method was based on the representation results for the distributions of exit times via Kolmogorov parabolic equations, and the estimates obtained included as a parameter the maximum of the first derivative of the solution of the Kolmogorov equation for expected exit time. For non-Markov processes, this approach is not applicable by the following reasons.

First of all, the corresponding representation results are available only for non-Markov processes in the entire space (see, e.g., Rozovkii [6]; the case of first exit time from domains with boundary is not covered so far. Let us explain why this is difficult. For the Markov case of diffusion processes, the representation results follow from Itô formula applied to the solutions of Kolmogorov equations in the domain. It is possible because usually these solutions are smooth enough thanks to classical regularity results for deterministic parabolic equation.

For non-Markov Itô processes, the corresponding analogs of Kolmogorov equations are backward stochastic partial differential equations, more precisely, backward parabolic ItôBismut equations. One needs again a priori certain smoothness for their solutions to apply Itô-Ventsell formula which is the appropriate replacement of Itô formula (see, e.g., Rozovskii [6]). However, the known results about regularity of the solution of backward SPDEs are insufficient for the case of domains with boundary. Respectively, the representation result was not obtained for first exit times of non-Markov processes.

Moreover, it is difficult to expect that the solutions of the backward SPDE have the first derivatives bounded as was required for the estimates in Dokuchaev [1], and that the corresponding maximum can be estimated.

To overcome all these difficulties, we developed a different technique that covers nonMarkov Itô processes as well as Markov processes as a special case. First, the first exit of a $n$-dimensional non-Markov process is reduced to first exit of a scalar non-Markov process. Second, this scalar process is transformed to a Wiener process by measure change and time change. Then the technique from [1] is applied for the new scalar Markov process to obtain estimates for first exit times. This solution covers the cases when either $n=1$ or $D$ has a lacuna. The new estimates are different; they are slightly weaker than the ones from [1] and cover the case of finite horizon only. However, they still allow to establish that first exit times of two Itô processes depend regularly on their trajectories. Moreover, they are themselves useful even for the Markov case, since they do not require to solve the Kolmogorov equation in $D \subset \mathbf{R}^{n}$ for expected first exit time. In fact, this equation is replaced by a simple one dimensional equation which solution is estimated. 


\section{Some definitions}

Let $(\Omega, \mathcal{F}, \mathbf{P})$ be a standard probability space, and let $w(t)=\left(w_{1}(t), \ldots, w_{n}(t)\right)$ be a $n$ dimensional Wiener process. Let $D \subset \mathbf{R}^{n}$ be an open bounded domain. Let $a_{i}, i=1,2$, be random vectors with values in $D$, independent from $w(\cdot)$.

Let $\mathcal{F}_{t}$ be the filtration generated by the random vectors $w(s), s \leq t, a_{1}, a_{2}$.

Consider two $n$-dimensional Itô processes $y_{i}(t), i=1,2$, such that

$$
\left\{\begin{array}{l}
d y_{i}(t)=f_{i}(t) d t+\beta_{i}(t) d w(t), \quad t>0 \\
y_{i}(0)=a_{i} .
\end{array}\right.
$$

Here $f_{i}:[0,+\infty) \times \Omega \rightarrow \mathbf{R}^{n}$ and $\beta_{i}:[0,+\infty) \times \Omega \rightarrow \mathbf{R}^{n \times n}$ are random processes progressively measurable with respect to the filtration $\mathcal{F}_{t}$.

Note that we do not exclude the case when $y_{i}(t)$ is a solution of Itô equation, i.e., when $\left(f_{i}(t), \beta_{i}(t)\right)=F_{i}\left(y_{i}(t), t, \omega\right)$ for some functions $F_{i}: \mathbf{R}^{n} \times[0,+\infty) \times \Omega \rightarrow \mathbf{R}^{n} \times \mathbf{R}^{n \times n}, i=1,2$.

We denote Euclidean norm as $|\cdot|$, and $\bar{D}$ denotes the closure of a region $D$.

Let $r_{1}, r_{2} \in \mathbf{R}$ be such that $r_{1}<r_{2}$.

For the case when $n=1$, set $O \triangleq\left\{x \in \mathbf{R}: r_{1}<x<r_{2}\right\}$. For the case when $n>1$, we assume that $r_{1}>0$ and $O \triangleq\left\{x \in \mathbf{R}^{n}: r_{1}<|x|<r_{2}\right\}$, i.e., it is a spherical layer.

We assume that there exists a bijection $\phi: D \rightarrow O$ such that the processes $\widehat{y}_{i}(t) \triangleq$ $\phi\left(y_{i}(t)\right), i=1,2$, are such that there exist random processes $\widehat{f}_{i}:[0,+\infty) \times \Omega \rightarrow \mathbf{R}$ and $\widehat{\beta}_{i}=\left(\widehat{\beta}_{i 1}, \ldots, \widehat{\beta}_{i n}\right):[0,+\infty) \times \Omega \rightarrow \mathbf{R}^{1 \times n}$ that are progressively measurable with respect to $\mathcal{F}_{t}$, square integrable in $(t, \omega) \in[0, T] \times \Omega$, and such that

$$
\left\{\begin{array}{l}
d r_{i}(t)=\widehat{f}_{i}(t) d t+\widehat{\beta}_{i}(t) d w(t), \quad t>0 \\
r_{i}(0)=\widehat{r}_{i}
\end{array}\right.
$$

where $r_{i}(t)=\left|\widehat{y}_{i}(t)\right|$ and $\widehat{r}_{i} \triangleq\left|\phi\left(a_{i}\right)\right| \in O$ for all $\omega$.

Further, we assume that $\left|\widehat{\beta}_{i}(t)\right|^{2} \in\left[c_{1}, c_{2}\right]$ and $\widehat{\beta}_{i 1}(t)^{2} \geq c_{3}$ for a.e. $t, \omega, i=1,2$, where $c_{k}>0$ are constants, $k=1,2,3, c_{1}<c_{2}$.

The conditions listed above cover two cases:

- $n=1$, and $D$ is a connected interval; or

- $n>1$, and $D \triangleq D_{1} \backslash D_{0}$, where $D_{i} \subset \mathbf{R}^{n}$ are domains with $C^{2}$-smooth boundaries $\partial D_{i}$, $i=0,1$, such that $D_{0} \subset D_{1}$ and $\partial D_{0} \cap \partial D_{1}=\emptyset$. In other words, there is a lacuna $D_{0}$ in the domain $D$. 
Note that, in the both cases, there exists a bijection $\phi: D \rightarrow O$ such that $\phi$ is continuously twice differentiable inside $D$, and the derivatives are uniformly bounded. The verification of the conditions required is straightforward.

We shall use the notation $x \wedge y \triangleq \min (x, y)$.

Let $t_{H}>0$ be given and non-random. Let

$$
\tau_{i} \triangleq t_{H} \wedge \inf \left\{t \geq 0: y_{i}(t) \notin D\right\}, \quad \tau_{\wedge} \triangleq \tau_{1} \wedge \tau_{2}
$$

Set $h_{i}(t) \triangleq \widehat{f}_{i}(t) \widehat{\beta}_{i 1}(t)^{-1}$.

Let $T_{*}$ be a non-random number such that $T_{*} \geq \operatorname{ess}^{\sup _{i=1,2, \omega \in \Omega}}\left(\tau_{\wedge}+\int_{\tau_{\wedge}}^{t_{H}}\left|\widehat{\beta}_{i}(t)\right|^{2} d t\right)$.

Let $\widehat{t}_{H} \geq t_{H}$ be a non-random time such that

$$
\operatorname{essinf} \sin _{i, 2, \omega \in \Omega}\left(\tau_{\wedge}+\int_{\tau_{\wedge}}^{\widehat{t}_{H}}\left|\widehat{\beta}_{i}(t)\right|^{2} d t\right) \geq T_{*} .
$$

Clearly, there exist numbers $T_{*}$ and $\widehat{t}_{H}$ with these properties.

Set $h_{i}(t) \triangleq \widehat{f}_{i}(t) \widehat{\beta}_{i 1}(t)^{-1}$. We assume that

$$
\mathbf{E} \exp \left(\frac{1}{2} \int_{0}^{\widehat{t}_{H}} h_{i}(t)^{2} d t\right)<+\infty .
$$

We denote by $\mathbb{I}$ the indicator function.

\section{The results}

Set $K \triangleq\left(r_{2}-r_{1}\right) \operatorname{ess} \sup _{i, t, \omega}\left|\widehat{\beta}_{i}(t, \omega)\right|^{-2}$.

Theorem 1 There exists a probability measure $Q$ being equivalent to the measure $\mathbf{P}$ and such that

$$
\mathbf{E}_{Q}\left|\tau_{1}-\tau_{2}\right| \leq K \mathbf{E}_{Q} \mathbb{I}_{\left\{\tau_{1} \neq \tau_{2}\right\}}\left|r_{1}\left(\tau_{\wedge}\right)-r_{2}\left(\tau_{\wedge}\right)\right|,
$$

where $\mathbf{E}_{Q}$ is the corresponding expectation.

Remark 1 An admissible measure $Q$ is constructed in the proof of Theorem 1 via $h_{i}$. In particular, this $Q$ is such that $Q=\mathbf{P}$ if $\widehat{f}_{1} \equiv \widehat{f}_{2} \equiv 0$, i.e., when the processes $r_{i}(t)$ are martingales.

Corollary 1 Assume that $\widehat{f}_{1} \equiv \widehat{f}_{2} \equiv 0$ and that the assumptions of Theorem 1 are satisfied. Then

$$
\mathbf{E}\left|\tau_{1}-\tau_{2}\right| \leq K \mathbf{E} \mathbb{I}_{\left\{\tau_{1} \neq \tau_{2}\right\}}\left|r_{1}\left(\tau_{\wedge}\right)-r_{2}\left(\tau_{\wedge}\right)\right| .
$$


If, in addition, $\widehat{\beta}_{1} \equiv \widehat{\beta}_{2}$, then

$$
\mathbf{E}\left|\tau_{1}-\tau_{2}\right| \leq K \mathbf{E}\left|r_{1}(0)-r_{2}(0)\right|
$$

Note that $K$ does note depend on $T$ and on the mutual distribution of $\widehat{\beta}, \widehat{f}, w$.

Since the construction of $Q$ in the proof of Theorem 1 is cumbersome for $h_{1} \neq h_{2}$, Theorem 1 is not very convenient to use in this case. However, this theorem helps to obtain the estimate under the original probability measure.

For $q>1$ and $m= \pm 1$, set

$$
\begin{aligned}
& C(q, m) \triangleq C_{1}(q, m)+C_{2}(q, m), \\
& C_{i}(q, m) \triangleq \widehat{t}_{\omega \in \Omega} \operatorname{ess} \sup \exp \left[\frac{q-m}{2} \int_{0}^{\widehat{t}_{H}} h_{i}(t)^{2} d t\right] .
\end{aligned}
$$

Theorem 2 Let $q>1$ be such that $C(q, m)<+\infty$ for $m= \pm 1$. Then

$$
\mathbf{E}\left|\tau_{1}-\tau_{2}\right| \leq t_{H}^{1 / q} K^{1 / p} C(q,-1) C(q, 1)^{1 / p}\left(\mathbf{E} \mathbb{I}_{\left\{\tau_{1} \neq \tau_{2}\right\}}\left|r_{1}\left(\tau_{\wedge}\right)-r_{2}\left(\tau_{\wedge}\right)\right|^{p}\right)^{1 / p^{2}},
$$

where $p=q(q-1)^{-1}$.

Remark 2 Estimate (4) implies that

$$
\mathbf{E}\left|\tau_{1}-\tau_{2}\right| \leq t_{H}^{1 / q} K^{1 / p} C(q,-1) C(q, 1)^{1 / p}\left(\mathbf{E} \sup _{t \in\left[0, t_{H}\right]}\left|r_{1}(t)-r_{2}(t)\right|^{p}\right)^{1 / p^{2}} .
$$

This last estimate is easier to apply, since $\mathbf{E} \sup _{t \in\left[0, t_{H}\right]}\left|r_{1}(t)-r_{2}(t)\right|^{p}$ can be estimated in many cases via certain norms of $r_{1}(0)-r_{2}(0), \widehat{f}_{1}-\widehat{f}_{2}$, and $\widehat{\beta}_{1}-\widehat{\beta}_{2}$ (see, e.g., Theorem 3.15 from Gikhman and Skorohod [3], or Theorem 2.8.7 from Krylov [4]). Similar modification can be done for estimate (3).

Remark 3 The estimates for Markov case from [1] correspond to the case when $p=1$ in (4), i.e., they are stronger than (4) in this sense. However, new estimates (4) are themselves useful even for the special case of Markov processes, since they do not require to solve ndimensional Kolmogorov equations for expected first exit time. In contrast, the estimates in [1] require the solution of the parabolic Kolmogorov equation in $D \subset \mathbf{R}^{n}$ for expected first exit time.

Remark 4 Estimate (4) uses the distributions of the processes $h_{i}$ after the original terminal time $t_{H}$. In contrast, the estimates from [1] use the distributions of parameters before terminal time only. 


\section{Proofs}

Proof of Theorem 1. Clearly, $\tau_{\wedge}$ is a Markov time with respect to the filtration $\mathcal{F}_{t}$. Set

$$
Z_{i} \triangleq \exp \left(-\int_{\tau_{\wedge}}^{\widehat{t}_{H}} h_{i}(t) d w_{1}(t)-\frac{1}{2} \int_{\tau_{\wedge}}^{\widehat{t}_{H}} h_{i}(t)^{2} d t\right) .
$$

By the assumptions, Novikov condition is satisfied, $\mathbf{E} Z_{i}=1$, and $\mathbf{E}\left\{Z_{i} \mid \mathcal{F}_{\tau_{\curlywedge}}\right\}=1$ a.s.. Therefore, we can define probability measures $Q_{i}$ such that $\frac{d Q_{i}}{d \mathbf{P}}=Z_{i}$.

Clearly,

$$
r_{i}(t)=r_{i}\left(\tau_{\wedge}\right)+\int_{\tau_{\wedge}}^{t} \widehat{\beta}_{i}(t) d w_{i}^{*}(t),
$$

where the processes $w_{i}^{*}(t)=\left(w_{i 1}^{*}(t), \ldots, w_{i n}^{*}(t)\right)$ are defined for $t>\tau_{\wedge}$ as

$$
w_{i 1}^{*}(t) \triangleq w_{1}\left(\tau_{\wedge}\right)+\int_{\tau_{\wedge}}^{t} h_{i}(s) d s, \quad w_{i k}^{*}(t) \triangleq w_{k}(t), \quad k=2, \ldots, n, \quad i=1,2 .
$$

By Girsanov Theorem, $w_{i}^{*}(t)$ is a Wiener process under $Q_{i}$ for $t \in\left[\tau_{\wedge}, \widehat{t}_{H}\right]$ on the conditional probability space given $\mathcal{F}_{\tau_{\wedge}}$, i.e., under the conditional measure $Q_{i}\left(\cdot \mid \mathcal{F}_{\tau_{\wedge}}\right), i=1,2$.

Set $M_{i}(t) \triangleq \int_{\tau_{\wedge}}^{t} \widehat{\beta}_{i}(s) d w_{i}^{*}(s), t \in\left[\tau_{\wedge}, \widehat{t}_{H}\right]$. Clearly, $M_{i}(t)$ is a vanishing at $\tau_{\wedge}$ martingale under the conditional measure $Q_{i}\left(\cdot \mid \mathcal{F}_{\tau_{\wedge}}\right)$ for $t \in\left[\tau_{\wedge}, \widehat{t}_{H}\right]$ with quadratic variation process

$$
\left[M_{i}\right](t) \triangleq \int_{\tau_{\wedge}}^{t}\left|\widehat{\beta}_{i}(s)\right|^{2} d s .
$$

Let $\theta_{i}(t) \triangleq\left[M_{i}\right]^{-1}\left(t-\tau_{\wedge}\right)$ be the inverse function, i.e.,

$$
\theta_{i}(t)=\inf \left\{s \geq \tau_{\wedge}:\left[M_{i}\right](s)=t-\tau_{\wedge}\right\}, \quad t=\tau_{\wedge}+\int_{\tau_{\wedge}}^{\theta_{i}(t)}\left|\widehat{\beta}_{i}(s)\right|^{2} d s .
$$

Note that $\theta_{i}\left(\tau_{\wedge}\right)=\tau_{\wedge}$, and the process $\theta_{i}(t)$ is strictly increasing in $t>\tau_{\wedge}$.

Let $T_{*}$ be the non-random number defined above, i.e., such that $T_{*} \geq \operatorname{ess}^{\sup _{i=1,2, \omega \in \Omega}}\left(\left[M_{i}\right]\left(t_{H}\right)+\right.$ $\left.\tau_{\wedge}\right)$.

By Dambis-Dubins-Schwarz Theorem (see, e.g., Revuz and Yor [5]), the process $B_{i}(t) \triangleq$ $M_{i}\left(\theta_{i}(t)\right)$ is a Brownian motion for $t \in\left[\tau_{\wedge}, T_{*}\right]$ under the conditional probability measure $Q_{i}\left(\cdot \mid \mathcal{F}_{\tau_{\wedge}}\right)$, and $M_{i}(t)=B_{i}\left(\left[M_{i}\right](t)\right)$. This process $B_{i}(t)$ is vanishing at $t=\tau_{\wedge}$

Let $z_{i}(t) \triangleq r_{i}(t)$ for $t \leq \tau_{\wedge}$, and $z_{i}(t) \triangleq r_{i}\left(\tau_{\wedge}\right)+B_{i}(t)$ for $t>\tau_{\wedge}$.

Set

$$
T_{i} \triangleq T_{*} \wedge \inf \left\{t \geq \tau_{\wedge}: z_{i}(t) \notin\left(r_{1}, r_{2}\right)\right\} .
$$

By the definitions, it follows that if $\tau_{\wedge}<t_{H}$, then $T_{i}=T_{*} \wedge\left(\tau_{\wedge}+\left[M_{i}\right]\left(\tau_{i}\right)\right)$ and $\tau_{\wedge}=T_{1} \wedge T_{2}$. In addition, it follows from the definitions that

$$
\begin{array}{ll}
\left\{\tau_{1}<\tau_{2}\right\} \subseteq\left\{T_{1}<T_{2}\right\}, & \left\{T_{1}<T_{2}\right\} \subseteq\left\{\tau_{1} \leq \tau_{2}\right\} \\
\left\{\tau_{2}<\tau_{1}\right\} \subseteq\left\{T_{2}<T_{1}\right\}, & \left\{T_{2}<T_{1}\right\} \subseteq\left\{\tau_{2} \leq \tau_{1}\right\}
\end{array}
$$


Let $\mathcal{G}_{i, t}$ be the filtration generated by $z_{i}(t)$. It follows that $\tau_{\wedge}$ is a Markov time with respect to the both filtrations $\mathcal{G}_{i, t}, i=1,2$.

Let

$$
e_{1} \triangleq \mathbb{I}_{\left\{\tau_{1}>\tau_{2}\right\}}, \quad e_{2} \triangleq \mathbb{I}_{\left\{\tau_{2}>\tau_{1}\right\}}
$$

We have that $e_{1} \leq \mathbb{I}_{\left\{T_{1}>T_{2}\right\}}, e_{2} \leq \mathbb{I}_{\left\{T_{2}>T_{1}\right\}}$.

Let the function $u:\left[r_{1}, r_{2}\right] \times\left(-\infty, T_{*}\right] \rightarrow \mathbf{R}$ be the solution of the problem

$$
\frac{\partial u}{\partial t}(x, t)+\frac{1}{2} \frac{\partial^{2} u}{\partial x^{2}}(x, t)=-1, \quad u\left(r_{1}, t\right)=u\left(r_{2}, t\right)=0, \quad u\left(x, T_{*}\right)=0 .
$$

Clearly, $u\left(z_{i}\left(T_{i}\right), T_{i}\right)=0, i=1,2$, and if $e_{1} \neq 0$, then $\tau_{1}>\tau_{2}$ and $T_{1}>T_{2}$. Similarly to [1], we obtain by Itô formula that

$$
\begin{array}{r}
\mathbf{E}_{Q_{1}}\left\{e_{1}\left\{u\left(z_{1}\left(T_{2}\right), T_{2}\right)-u\left(z_{2}\left(T_{2}\right), T_{2}\right)\right\}\right\}=-\mathbf{E}_{Q_{1}}\left\{e_{1}\left\{u\left(z_{1}\left(T_{1}\right), T_{1}\right)-u\left(z_{1}\left(T_{2}\right), T_{2}\right)\right\}\right\} \\
=-\mathbf{E}_{Q_{1}} e_{1} \int_{\tau_{\wedge}}^{T_{1}}\left(\frac{\partial u}{\partial t}\left(z_{1}(t), t\right)+\frac{1}{2} \frac{\partial^{2} u}{\partial x^{2}}\left(z_{1}(t), t\right)\right) d t=\mathbf{E}_{Q_{1}} e_{1}\left(T_{1}-\tau_{\wedge}\right) .
\end{array}
$$

We have used here that $e_{i}$ is measurable with respect to the $\sigma$-algebra $\mathcal{G}_{i, \tau_{\wedge}}$ associated with Markov time (with respect to $\mathcal{G}_{i, t}$ ) $\tau_{\wedge}$. It follows from the fact that $e_{i}=\mathbb{I}_{\left\{\tau_{i}>\tau_{\wedge}\right\}}, i=1,2$ (see, e.g., Gihman and Skorohod [2], Chapter 4, §2).

Let the function $v:\left[r_{1}, r_{2}\right] \rightarrow \mathbf{R}$ be defined as $v(x) \triangleq\left(x-r_{1}\right)\left(r_{2}-x\right)$. We have that $v^{\prime \prime}(x)=-2, v\left(r_{1}\right)=v\left(r_{2}\right)=0$.

Let $W_{s}(t)$ be a standard Wiener process for $t \geq s, W_{s}(s)=0$. Let

$$
T^{x, s} \triangleq \inf \left\{t \geq s: x+W_{s}(t) \notin\left(r_{1}, r_{2}\right)\right\}, \quad x \in\left(r_{1}, r_{2}\right) .
$$

It follows from the classical theory of Markov processes that

$$
v(x)=\mathbf{E} T^{x, 0}, \quad u(x, s)=\mathbf{E}\left(T^{x, s} \wedge T_{*}\right)=\mathbf{E}\left(T^{x, 0} \wedge\left(T_{*}-s\right)\right) .
$$

Hence

$$
0<u(x, t)<v(x), \quad u(x, t) \underset{t \rightarrow-\infty}{\rightarrow} v(x), \quad \forall x \in\left(r_{1}, r_{2}\right), \quad \frac{\partial u}{\partial t}(x, t) \leq 0 .
$$

Further, it follows that

$$
0 \geq \frac{\partial^{2} u}{\partial x^{2}}(x, t)=-2-2 \frac{\partial u}{\partial t}(x, t) \geq-2=\frac{\partial^{2} v}{\partial x^{2}}(x),
$$

and that

$$
\frac{u(x, t)-u\left(r_{1}, t\right)}{x-r_{1}} \leq \frac{v(x)-v\left(r_{1}\right)}{x-r_{1}}, \quad \frac{u\left(r_{2}, t\right)-u(x, t)}{r_{2}-x} \geq \frac{v\left(r_{2}\right)-v(x)}{r_{2}-x}, \quad x \in\left(r_{1}, r_{2}\right) .
$$


Hence

$$
0 \leq \frac{\partial u}{\partial x}\left(r_{1}, t\right) \leq \frac{\partial v}{\partial x}\left(r_{1}\right), \quad 0 \geq \frac{\partial u}{\partial x}\left(r_{2}, t\right) \geq \frac{\partial v}{\partial x}\left(r_{2}\right)
$$

In addition, we have that

$$
\frac{\partial u}{\partial x}(x, t)=\frac{\partial u}{\partial x}\left(r_{1}, t\right)+\int_{r_{1}}^{x} \frac{\partial^{2} u}{\partial x^{2}}(z, t) d z \geq 0, \quad \frac{\partial v}{\partial x}(x)=\frac{\partial v}{\partial x}\left(r_{1}\right)+\int_{r_{1}}^{x} \frac{\partial^{2} v}{\partial x^{2}}(z) d z \geq 0
$$

for $x \in\left(r_{1},\left(r_{1}+r_{2}\right) / 2\right)$, and

$$
\frac{\partial u}{\partial x}(x, t)=\frac{\partial u}{\partial x}\left(r_{2}, t\right)-\int_{x}^{r_{2}} \frac{\partial^{2} u}{\partial x^{2}}(z, t) d z \leq 0, \quad \frac{\partial v}{\partial x}(x)=\frac{\partial v}{\partial x}\left(r_{2}\right)-\int_{x}^{r_{2}} \frac{\partial^{2} v}{\partial x^{2}}(z) d z \leq 0
$$

for $x \in\left(\left(r_{1}+r_{2}\right) / 2, r_{2}\right)$. Hence

$$
\sup _{x \in\left(r_{1}, r_{2}\right), t \geq 0}\left|\frac{\partial u}{\partial x}(x, t)\right| \leq K_{v}, \quad \text { where } K_{v} \triangleq \sup _{x \in\left(r_{1}, r_{2}\right)}\left|\frac{d v}{d x}(x)\right| .
$$

Clearly, $v^{\prime}(x)=r_{2}+r_{1}-2 x$, and

$$
K_{v}=r_{2}-r_{1}
$$

By (5)-(6),

$$
\mathbf{E}_{Q_{1}} e_{1}\left(T_{1}-\tau_{\wedge}\right) \leq K_{v} \mathbf{E}_{Q_{1}} e_{1}\left|z_{1}\left(\tau_{\wedge}\right)-z_{2}\left(\tau_{\wedge}\right)\right|=K_{v} \mathbf{E}_{Q_{1}} e_{1}\left|r_{1}\left(\tau_{\wedge}\right)-r_{2}\left(\tau_{\wedge}\right)\right| .
$$

Let $K_{\beta} \triangleq \operatorname{ess}_{\sup } \operatorname{su}_{i, \omega}\left|\widehat{\beta}_{i}(t, \omega)\right|^{-2}$.

Assume that $e_{1}>0$, i.e., $\tau_{1}>\tau_{2}$. It follows that $\tau_{\wedge}<t_{H}$. Hence $T_{1}=\left[M_{1}\right]\left(\tau_{1}\right)+\tau_{\wedge}$. Then

$$
\tau_{1}=\tau_{\wedge}+\int_{\tau_{\wedge}}^{\tau_{1}}\left|\widehat{\beta}_{1}(s)\right|^{-2}\left|\widehat{\beta}_{1}(s)\right|^{2} d s \leq \tau_{\wedge}+K_{\beta} \int_{\tau_{\wedge}}^{\tau_{1}}\left|\widehat{\beta}_{1}(s)\right|^{2} d s=\tau_{\wedge}+K_{\beta}\left(T_{1}-\tau_{\wedge}\right)
$$

and

$$
\left|\tau_{1}-\tau_{\wedge}\right| \leq K_{\beta}\left|T_{1}-\tau_{\wedge}\right|
$$

It follows that

$$
e_{1}\left(\tau_{1}-\tau_{\wedge}\right) \leq e_{1} K_{\beta}\left|T_{1}-\tau_{\wedge}\right|
$$

By (7)-(8), it follows that

$$
\mathbf{E}_{Q_{1}} e_{1}\left|\tau_{1}-\tau_{\wedge}\right| \leq K_{\beta} \mathbf{E}_{Q_{1}} e_{1}\left|T_{1}-\tau_{\wedge}\right| \leq K_{v} K_{\beta} \mathbf{E}_{Q_{1}} e_{1}\left|r_{1}\left(\tau_{\wedge}\right)-r_{2}\left(\tau_{\wedge}\right)\right|
$$

If we replaced the indices 1,2 in (9) by 2,1 , we get an analogous expression for $\mathbf{E}\left\{e_{2}\left(\tau_{2}-\right.\right.$ $\left.\left.\tau_{\wedge}\right)\right\}:$

$$
\mathbf{E}_{Q_{2}} e_{2}\left(\tau_{2}-\tau_{\wedge}\right) \leq K_{v} K_{\beta} \mathbf{E}_{Q_{2}} e_{2}\left|r_{1}\left(\tau_{\wedge}\right)-r_{2}\left(\tau_{\wedge}\right)\right|
$$


Combining (9) and (10), we obtain that

$$
\begin{aligned}
& \mathbf{E}_{Q_{1}} e_{1}\left|\tau_{1}-\tau_{\wedge}\right|+\mathbf{E}_{Q_{2}} e_{2}\left|\tau_{2}-\tau_{\wedge}\right| \\
& \leq K_{v} K_{\beta}\left[\mathbf{E}_{Q_{1}} e_{1}\left|r_{1}\left(\tau_{\wedge}\right)-r_{2}\left(\tau_{\wedge}\right)\right|+\mathbf{E}_{Q_{2}} e_{2}\left|r_{1}\left(\tau_{\wedge}\right)-r_{2}\left(\tau_{\wedge}\right)\right|\right] .
\end{aligned}
$$

Set $e_{0} \triangleq \mathbb{I}_{\left\{\tau_{1}=\tau_{2}\right\}}=1-e_{1}-e_{2}$ and $\widehat{Z} \triangleq e_{0}+e_{1} Z_{1}+e_{2} Z_{2}$. We have that $\kappa \triangleq \mathbf{E} \widehat{Z} \in(0,+\infty)$. Set $Z \triangleq \kappa^{-1} \widehat{Z}$. Introduce the probability measure $Q$ such that $\frac{d Q}{d P}=Z$. Since $\widehat{Z}(\omega)>0$ for all $\omega$, this measure is equivalent to the original measure $\mathbf{P}$. Note that $\mathbf{E}_{Q} e_{i} \xi=\kappa^{-1} \mathbf{E}_{Q_{i}} e_{i} \xi$ for any integrable random variable $\xi, i=1,2$.

By (11), it follows that

$$
\mathbf{E}_{Q}\left|\tau_{1}-\tau_{2}\right| \leq K_{v} K_{\beta} \mathbf{E}_{Q} \mathbb{I}_{\left\{\tau_{1} \neq \tau_{2}\right\}}\left|r_{1}\left(\tau_{\wedge}\right)-r_{2}\left(\tau_{\wedge}\right)\right|
$$

Now the assertion of Theorem 1 follows at once from (12) with $K=K_{v} K_{\beta}$.

Proof of Theorem 2. Let $Z_{i}, Z, e_{i}$, and $Q$, be defined in the proof of Theorem 1. By the definitions and Itô formula, it follows that $Z_{i}^{m}=\eta_{i, m}\left(\widehat{t}_{H}\right)$, where $m= \pm 1$,

$$
\begin{aligned}
& d \eta_{i, 1}(t)=-h_{i}(t) \eta_{i, 1}(t) d w_{1}(t), \quad \eta_{i, 1}\left(\tau_{\wedge}\right)=1, \\
& d \eta_{i,-1}(t)=h_{i}(t)^{2} \eta_{i,-1}(t) d t+h_{i}(t) \eta_{i,-1}(t) d w_{1}(t), \quad \eta_{i,-1}\left(\tau_{\wedge}\right)=1 .
\end{aligned}
$$

It follows that $Z_{i}^{m q}=\zeta_{i, q, m}\left(\widehat{t}_{H}\right)$ for $m= \pm 1$, where the process $\zeta_{i, q, m}(t)$ is the solution of

$$
\begin{aligned}
& d \zeta_{i, q, m}(t)=\left[q \frac{1-m}{2}+\frac{q(q-1)}{2}\right] h_{i}(t)^{2} \zeta_{i, q, m}(t) d t-q m h_{i}(t) \zeta_{i, q, m}(t) d w_{1}(t), \\
& \zeta_{i, q, m}\left(\tau_{\wedge}\right)=1
\end{aligned}
$$

Note that $q \frac{1-m}{2}+\frac{q(q-1)}{2}=q \frac{q-m}{2}$ for $m= \pm 1$. It follows that $\mathbf{E} Z_{i}^{m q}=\mathbf{E} \zeta_{i, q, m}\left(\widehat{t}_{H}\right) \leq C_{i}(q, m)$, $m= \pm 1$. We obtain this by observing that if $x(t)$ is the solution of a linear Itô equation $d x(t)=x(t)\left[\alpha(t) d t+\sigma(t) d w_{1}(t)\right]$, where $\alpha(t) \geq 0$ and $\sigma(t)$ are square integrable $\mathcal{F}_{t^{-}}$adapted processes, and $x\left(\tau_{\wedge}\right)=1$, then

$$
\begin{aligned}
& \mathbf{E} x(t)=\mathbf{E} \exp \int_{\tau_{\wedge}}^{t} \alpha(s) d s \cdot \exp \int_{\tau_{\wedge}}^{t}\left(-\frac{\sigma(s)^{2}}{2} d s+\sigma(s) d w_{1}(s)\right) \\
& \leq \underset{\omega}{\operatorname{ess} \sup \exp } \int_{0}^{t} \alpha(s) d s \mathbf{E} \exp \int_{0}^{t}\left(-\frac{\sigma(s)^{2}}{2} d s+\sigma(s) d w_{1}(s)\right)=\underset{\omega}{\operatorname{ess} \sup \exp } \int_{0}^{t} \alpha(s) d s .
\end{aligned}
$$

Further,

$$
\begin{aligned}
& Z^{m q}=\left(e_{0}+e_{1} Z_{1}+e_{2} Z_{2}\right)^{m q}=e_{0}+e_{1} Z_{1}^{m q}+e_{2} Z_{2}^{m q}, \\
& e_{0} \mathbb{I}_{\left\{\tau_{1} \neq \tau_{2}\right\}}=0, \quad e_{1} \mathbb{I}_{\left\{\tau_{1} \neq \tau_{2}\right\}}=e_{1}, \quad e_{2} \mathbb{I}_{\left\{\tau_{1} \neq \tau_{2}\right\}}=e_{2} .
\end{aligned}
$$


Hence

$$
\left(\mathbf{E} \mathbb{I}_{\left\{\tau_{1} \neq \tau_{2}\right\}} Z^{m q}\right)^{1 / q} \leq\left(\mathbf{E} Z_{1}^{m q}\right)^{1 / q}+\left(\mathbf{E} Z_{2}^{m q}\right)^{1 / q} \leq C(q, m)
$$

Further, we have that

$$
\begin{array}{r}
\mathbf{E}\left|\tau_{1}-\tau_{2}\right|=\mathbf{E}_{Q} \mathbb{I}_{\left\{\tau_{1} \neq \tau_{2}\right\}} Z^{-1}\left|\tau_{1}-\tau_{2}\right| \leq\left[\mathbf{E}_{Q} \mathbb{I}_{\left\{\tau_{1} \neq \tau_{2}\right\}} Z^{-q}\right]^{1 / q}\left[\mathbf{E}_{Q}\left|\tau_{1}-\tau_{2}\right|^{p}\right]^{1 / p} \\
\leq C(q,-1)\left[\mathbf{E}_{Q}\left|\tau_{1}-\tau_{2}\right|^{p}\right]^{1 / p}
\end{array}
$$

Since $\left|\tau_{1}-\tau_{2}\right| \leq t_{H}$, we have that

$$
\mathbf{E}_{Q}\left|\tau_{1}-\tau_{2}\right|^{p} \leq t_{H}^{p-1} \mathbf{E}_{Q}\left|\tau_{1}-\tau_{2}\right|
$$

Remind that $Q$ is the same measure as in the proof of Theorem 1, and this theorem holds for this measure. It follows that

$$
\begin{aligned}
& \mathbf{E}_{Q}\left|\tau_{1}-\tau_{2}\right|^{p} \leq t_{H}^{p-1} K \mathbf{E}_{Q} \mathbb{I}_{\left\{\tau_{1} \neq \tau_{2}\right\}}\left|r_{1}\left(\tau_{\wedge}\right)-r_{2}\left(\tau_{\wedge}\right)\right|=t_{H}^{p-1} K \mathbf{E} \mathbb{I}_{\left\{\tau_{1} \neq \tau_{2}\right\}} Z\left|r_{1}\left(\tau_{\wedge}\right)-r_{2}\left(\tau_{\wedge}\right)\right| \\
& \leq t_{H}^{p-1} K\left(\mathbf{E} \mathbb{I}_{\left\{\tau_{1} \neq \tau_{2}\right\}} Z^{q}\right)^{1 / q}\left(\mathbf{E} \mathbb{I}_{\left\{\tau_{1} \neq \tau_{2}\right\}}\left|r_{1}\left(\tau_{\wedge}\right)-r_{2}\left(\tau_{\wedge}\right)\right|^{p}\right)^{1 / p} \\
& \leq t_{H}^{p-1} K C(q, 1)\left(\mathbf{E} \mathbb{I}_{\left\{\tau_{1} \neq \tau_{2}\right\}}\left|r_{1}\left(\tau_{\wedge}\right)-r_{2}\left(\tau_{\wedge}\right)\right|^{p}\right)^{1 / p} .
\end{aligned}
$$

Hence

$$
\left[\mathbf{E}_{Q}\left|\tau_{1}-\tau_{2}\right|^{p}\right]^{1 / p} \leq t_{H}^{\frac{p-1}{p}} K^{1 / p} C(q, 1)^{1 / p}\left(\mathbf{E} \mathbb{I}_{\left\{\tau_{1} \neq \tau_{2}\right\}}\left|r_{1}\left(\tau_{\wedge}\right)-r_{2}\left(\tau_{\wedge}\right)\right|^{p}\right)^{1 / p^{2}}
$$

Clearly, $1 / q=(p-1) / p$. Combining (12) and (13), we obtain (4). This completes the proof of Theorem 2.

\section{Example}

The choice of the bijection $\phi$ for an admissible domain $D$ is rather a geometrical problem. For a given $\phi$, the coefficients of Itô equation for the corresponding process $\widehat{y}(t)=\phi(y(t))$ can be found immediately using Itô formula. Similarly, the coefficients $\widehat{f}$ and $\widehat{\beta}$ can be found from Itô formula again applied for the function $r(t)=|\widehat{y}(t)|$ which is smooth in $O=\phi(D)$. We leave this part without an example.

We illustrate how to calculate the constants in the estimates for given $\widehat{f}$ and $\widehat{\beta}$ on the following example.

We assume that (2) holds with $\widehat{f}_{1} \equiv \widehat{f}_{2} \equiv \widehat{f}$ and $\widehat{\beta}_{1} \equiv \widehat{\beta}_{2} \equiv \widehat{\beta}$, where $\widehat{f}$ and $\widehat{\beta}=$

$\left(\widehat{\beta}_{1}, \ldots, \widehat{\beta}_{n}\right)$ are some bounded functions progressively measurable with respect to $\mathcal{F}_{t}$. In addition, we assume that $\widehat{r}_{i}=r_{i}(0) \in\left(r_{1}, r_{2}\right)$ are non-random numbers, and that $r_{2}-r_{1}=1$, 
$|\widehat{\beta}(s)|^{2} \in\left[K_{-}, K_{+}\right]$, where $0<K_{-} \leq K_{+}<+\infty$ are constants, $K_{-}=K_{\beta}^{-1}$. In that case, $r_{1}(t)-r_{2}(t) \equiv r_{1}(0)-r_{2}(0), K_{v}=\sup |d v(x) / d x|=1, K=K_{\beta}=K_{-}^{-1}$, and $Q_{1}=Q_{2}=Q$.

Since $|\widehat{\beta}(t)|^{2} \in\left[K_{-}, K_{+}\right]$, we can take $T_{*} \triangleq t_{H} \max \left(1, K_{+}\right)$and $\widehat{t}_{H} \triangleq t_{H} \frac{\max \left(1, K_{+}\right)}{\min \left(1, K_{-}\right)}$.

By Theorem 1, we have

$$
\mathbf{E}_{Q}\left|\tau_{1}-\tau_{2}\right| \leq K_{\beta}\left|r_{1}(0)-r_{2}(0)\right| \quad \forall t_{H}>0
$$

First, let us assume that $\widehat{f} \equiv 0$. In this case, $h_{i} \equiv 0$ for $i=1,2, Z=Z_{1}=Z_{2}=1$, and $Q=\mathbf{P}$. By Corollary 1 , we have that the following explicit estimate holds:

$$
\mathbf{E}\left|\tau_{1}-\tau_{2}\right| \leq K_{\beta}\left|r_{1}(0)-r_{2}(0)\right| \quad \forall t_{H}>0
$$

Second, let us assume that $\widehat{f}(\cdot) \neq 0$ and $|\widehat{f}(t, \omega)| \leq K_{f}$ for all $t, \omega$, where $K_{f}>0$ is a constant. Then $\left|h_{i}\right| \leq K_{h}$, where $K_{h} \triangleq K_{f} \sqrt{K_{\beta}}$. Clearly,

$$
C(q, m) \leq C_{*}(q, m), \quad m= \pm 1,
$$

where

$$
C_{*}(q, m) \triangleq 2 \exp \left(\frac{q-m}{2} K_{h}^{2} \widehat{t}_{H}\right) .
$$

By Theorem 2, it follows that

$$
\mathbf{E}\left|\tau_{1}-\tau_{2}\right| \leq t_{H}^{1 / q} K_{\beta}^{1 / p} C_{*}(q, 1) C_{*}(q,-1)^{1 / p}\left|r_{1}(0)-r_{2}(0)\right|^{1 / p}
$$

for any $q>1, t_{H}>0$, and $p=q(q-1)^{-1}$.

Remark 5 Note that the similar approach could be applied to n-dimensional vector nonMarkov Itô processes without change of the domain, if these processes can be transformed to Markov diffusion processes by some time change. In that case, domains without lacuna could be included. However, this time change is known to exist for special cases only, for instance, when the same time change can work for all components.

\section{References}

[1] Dokuchaev, N.G., 2004, Estimates for distances between first exit times via parabolic equations in unbounded cylinders. Probability Theory and Related Fields, 129 (2), 290314.

[2] Gihman, I.I., and Skorohod, A.V., 1975, The Theory of Stochastic Processes. Vol. 2. Springer-Verlag, New York. 
[3] Gikhman, I.I., and Skorohod, A.V., 1979, Controlled Stochastic Processes. SpringerVerlag, New York-Heidelberg.

[4] Krylov, N.V., 1980, Controlled Diffusion Processes. Berlin, Heidelberg, New York: Shpringer-Verlag.

[5] Revuz, D., and Yor, M., 1999, Continuous Martingales and Brownian Motion. SpringerVerlag: New York.

[6] Rozovskii, B.L., 1990, Stochastic Evolution Systems; Linear Theory and Applications to Non-Linear Filtering. Kluwer Academic Publishers. Dordrecht-Boston-London. 